\title{
Alternative staging of regional lymph nodes in gastric cancer
}

\author{
Antoni M. Szczepanik ${ }^{1}$, Agata Paszko ${ }^{1}$, Miroslaw Szura ${ }^{1}$, Thecla Scully-Horner ${ }^{2}$, Jan Kulig ${ }^{1}$ \\ ${ }^{1} 1^{\text {st }}$ Department of General, Gastrointestinal, and Oncologic Surgery, Jagiellonian University Medical College, Krakow, Poland \\ IIntensive Care Unit, Queen Alexandra Hospital, Portsmouth, United Kingdom
}

Gastroenterology Rev 2016; 11 (3): 145-149

DOI: $10.5114 / p g .2016 .61492$

Key words: gastric cancer, lymphadenectomy, lymph node staging.

Address for correspondence: Antoni M. Szczepanik MD, PhD, $1^{\text {st }}$ Department of General, Gastrointestinal, and Oncologic Surgery, Jagiellonian University Medical College, 40 Kopernika St, 31-501 Krakow, Poland, phone/fax: +48 1242480 07, e-mail: msszczep@cyf-kr.edu.pl

\begin{abstract}
The TNM pN stage based on the number of metastatic lymph nodes is an independent prognostic factor in gastric cancer. Many studies have highlighted the phenomenon of stage migration and problems in comparing groups of patients with different numbers of total lymph nodes harvested within TNM staging. The current version of UICC/AJCC and JGCA TNM classifications postulates a minimal number of 16 lymph nodes as the base for $\mathrm{N}$ stage determination. Alternative systems such as lymph node ratio (LNR), positive to negative lymph node ratio (PNLNR), and LOGODDS (or LODDS), were implemented to increase the quality of $\mathrm{LN}$ assessment. These methods have reached the background in the literature, but to date no standard approach according to the cut-offs for the stages has been implemented. LOGODDS is the method that most reflects the number of harvested lymph nodes. The rationale for alternative staging methods, their correlations, and limitations are presented.
\end{abstract}

\section{Introduction}

The need for the appropriate classification of the advancement of any cancer is clear. The postoperative pathologic staging of cancer should provide the clinician with information on which to base a prognosis and indications for further adjuvant treatment.

In order to obtain precise data and thereby a reliable background for a treatment plan, the staging systems have evolved. This situation is observed in gastric cancer staging. The most variable factor in gastric cancer staging is the status of lymph nodes (LN). The topographic classification was used in the $4^{\text {th }}$ edition of the UICC/ AJCC TNM classification and in the former Japanese JGCA staging system. From the $5^{\text {th }}$ UICC/AJCC , through the $6^{\text {th }}$ and current $7^{\text {th }}$ edition, the number of metastatic $L N$ is the base for staging [1]. The same method is adopted for the JGCA classification published in English in 2011 [2]. This is important for the comparison of Japanese and non-Japanese trial results and the common language of scientific discussion. The evolution of staging system indicates that there is no "ideal staging" for gastric cancer and many other cancers. Every update is criticised in a number of papers analysing some of the details [3]. On the other hand, UICC/AJCC and JGCA classifications require standard surgical treatment with the minimal number of $L N$ retrieved. Compliance with this rule in many investigated groups of patients is poor [4]. Therefore, studies on more accurate LN staging methods deal both with the group of adequate and inadequate number of $L N$ assessed.

The aim of this analysis is to compare different methods of LN staging adopted to assess patients with limited numbers of LN retrieved.

\section{The number of lymph nodes assessed}

Radical gastrectomy with D2 LN dissection is the standard in Japanese, NCCN (USA), and ESMO (Europe) guidelines and in the majority of national guidelines worldwide for the treatment of advanced gastric cancer [5]. The above-mentioned common guideline is the result of debate and randomised clinical trials, which showed no benefit in survival for extended lymphadenectomy (D3) when compared to D2, and the increase of survival with D2 compared to D1 [6]. Of importance are the acceptable mortality and morbidity rates of D2 lymphadenectomy in experienced centres 
[6]. But the problem is the compliance to guidelines and the groups of patients treated before the guidelines. In the well-known INT0116 trial performed in the USA, even if D2 lymphadenectomy was the standard for this trial, the rate of D2 was about 10\% [7]. Another study from the USA reported that at least $15 \mathrm{LN}$ were assessed in $38 \%$ of teaching hospitals and in $31 \%$ of community hospitals [4]. Better adherence to guidelines was reported from the UK, where $74 \%$ of radical gastrectomies fulfilled the standard of 15 LN [8]. Dutch guidelines set the number at $11 \mathrm{LN}$, as a minimal standard. In the first period of implementation of this standard $49 \%$ met the criteria, but in the second period it was $59 \%$ [9]. The definition of D2 lymphadenectomy itself does not specify the number of LN harvested, but according to the definition of JGCA, D2 includes 13 LN stations (by total gastrectomy) or $11 \mathrm{LN}$ stations (by subtotal, distal gastrectomy). Presumably, the number of LN should exceed the number of stations. Therefore the patients who had less than 13-16 nodes dissected are D1/D1+ rather than D2. On the other hand, the number of $L N$ dissected within specimens is not equal to the number of LN examined by the pathologist. The quality of pathologic examination is another limitation of the final LN stage assessment, and it may be poor [10]. Both UICC/AJCC and JGCA classifications have "soft" requirements according to the number of $L N$ harvested. TNM $7^{\text {th }}$ edition: "Histological examination of a regional lymphadenectomy specimen will ordinarily include 16 or more lymph nodes." [1].

JGCA 2012 edition: "Although it is not a prerequisite, the examination of 16 or more lymph nodes is recommended for N status determination." [2].

Another phenomenon is that in patients with a higher number of metastatic LN usually more LN are dissected by the surgeon and/or examined by the pathologist [11]. A paper from China, published in 2012 [12], analysed 435 node-negative patients after D2 lymphadenectomy. In this group the mean number of $L N$ in the specimen was $13.5 \pm 4.5$ and the range $1-53$. In 105 patients 1-6 LN were examined, and 16 or more LN were examined in 148 patients. The number of LN in the specimen may be diminished by preoperative chemotherapy or radiotherapy, or by previous surgery. Lower $L N$ numbers are also observed in the elderly. In the above-mentioned study all patients with preoperative chemo- or radiotherapy were excluded from the analysis. One should remember that $L N$ may have a small (3-4 mm) diameter, so proper identification of $L N$ in fatty tissue is difficult.

\section{Impact of numbers on survival}

The $\mathrm{pN}$ stage is an independent prognostic factor in gastric cancer [13]. However, many studies indicate the phenomenon of stage migration [14]. The clue is that the small number of LN harvested is correlated with the lower number of metastatic LN. Therefore, patients are classified in lower than proper pN-stage. Any patient with total of $6 \mathrm{LN}$ harvested cannot achieve TNM N3 stage. But some of them have another metastatic LN, which was not dissected or examined by the pathologist. In a group of proper (we do not know the exact number) LN examined some of the patients will move upwards in the stages ladder and the survival in lower stages will be better because of exclusion of underestimated pN stages. The more extended lymphadenectomy can move some patients to more advanced pN stages, but cannot move them to less advanced one. However, when we move a patient with lower than average for the stage " $x$ " survival to the stage " $x+1$ ", and the survival of this particular patient is higher than the average survival for the stage " $x+1$ ", this patient can improve the survival in both stages. This is the so-called Will Rogers phenomenon [15]. Such a situation was observed with the change of $6^{\text {th }}$ UICC/AJCC edition to $7^{\text {th }}$ edition (for example patients with three positive LN were moved from N1 to N2). The increase of the number of LN assessed makes stage migration, or enables proper classification with UICC/AJCC and JGCA system. This may be the reason why some authors reported better survival in subgroups of equally staged patients stratified by the total number of LN assessed or higher number of negative LN [16]. Also, the LN assessed as "negative" contain some cancer cells. By removal of negative, by the standard meaning, LN the radicality is changed from R1 to RO.

\section{Lymph nodes ratio}

Quite a simple idea is to improve the staging system of $L N$ by the implementation of the ratio of positive $L N$ to total number of $L N$ harvested. According to many authors this can decrease stage migration $[17,18]$. In this type of staging we operate with a kind of "probability" of LN metastasis. Two positive LN over four harvested is equal to $4 / 8,5 / 10$, etc. In the same lymph node ratio (LNR) there are patients assessed otherwise as pN1, pN2, pN3a, and pN3b. Nevertheless, the results of some studies are in favour of the LNR system [18]. However, there is no system. Almost every paper uses its own classification according to the ranges of LNR in subgroups (Table I) [19-22].

Some studies are performed mostly on groups of patients with low total LN count, some use groups with a mean number of LN harvested over 20 [20]. For the implementation of the idea of LNR into practice there is a need for common intervals (subgroups) and probably 
Table I. The cutoffs of the studies using LNR

\begin{tabular}{lcc}
\hline Author & Patients (mean LN number) & LNR cut-offs \\
\hline Pedrazzani et al. 2010 [20] & $526(5.6)$ & $0,0.1-0.25,>0.25$ \\
\hline Liu et al. 2007 [17] & $224(28.1)$ & $0,0.1-0.4,0.41-0.8,>0.8$ \\
\hline Wang et al. 2012 [18] & $18403(10-11)$ & $0-0.067,0.068-0.3,0.31-0.7,>0.7$ \\
\hline Lee et al. 2012 [21] & $370(37)$ & $0.0-0.01,0.02-0.05,0.06-0.1,0.11-0.20,0.20-0.30,>0.30$ \\
\hline Espin et al. 2012 [11] & $96(22-31)$ & $<0.2, \geq 0.2$ \\
\hline Huang et al. 2010 [22] & $634(23.1)$ & $<0.4,0.21-0.5,>0.5$ \\
\hline Kulig et al. 2009 [19] & $735(8)$ & 0.4
\end{tabular}

${ }^{1}$ Wang et al. use pNRO only for patients with more than 15 LN harvested.

the assessment of the impact of the total number of LN on survival within subgroups.

\section{Positive to negative lymph nodes and the LOGODDS}

There are also studies using another ratio, positive LN over negative LN (PNLNR). This is a mathematical transformation of the LNR.

For every patient of negative $\mathrm{LN}>0, a, a 1, a 2$, positive LN count, $b, b 1, b 2$ - negative LN count: LNR $=a /(a+b)$. When 2 patients have the same LNR: $a 1 /(a 1+b 1)=a 2 /(a 2+b 2) \leftrightarrow a 1(a 2+b 2)=a 2(a 1+b 1)$ $\leftrightarrow a 1 a 2+a 1 b 2=a 2 a 1+a 2 b 1 \leftrightarrow a 1 b 2=a 2 b 1 \leftrightarrow a 1 /$ $b 1=a 2 / b 2$.

Theses 2 patients have the same ratio of positive to negative $\mathrm{LN}$.

Therefore, the stratification by LNR will give the same results as stratification by PNLNR.

LOGODDS is, by the definition of the authors [23], the natural logarithm from the ratio of positive $\mathrm{LN}+$ 0.5 /negative $L N+0.5$.

The original formula is $\log \frac{(\text { pnod }+0.5)}{(\text { tnod }- \text { pnod }+0.5)}$; however "tnod - pnod" is the count of negative nodes and we will use the latter.*

The authors of the formula did not use just the logPNLNR, as they commented, "to avoid singularity". This is to avoid dividing by 0 , when all LN are positive, and to avoid the result $-\infty$ (minus infinity) when the count of positive $L N$ is 0 . But they did

\footnotetext{
* Note - with regard to using logarithms, reference is made to the "natural logarithm", that is, a logarithm to base e, which is usually denoted by $\ln (x)$; however, the formula log $\frac{(\text { pnod }+0.5)}{\text { (tnod }- \text { pnod }+0.5)}$ uses the notation "log", which is more often used for a logarithm to base 10; this does not materially affect how the formula works for means of comparison (although of course the answers for individual sets will differ) and using "log" does have the advantage of avoiding the confusion between the natural logarithm In and the abbreviation LN for lymph node.
}

not comment on other consequences of adding 0.5 . Without this the ratio $1 / 5$ is equal to $2 / 10$, which is in turn equal to $3 / 15$, etc. With +0.5 the ratio is $1 / 5$ $>2 / 10>3 / 15$, etc. Consequently, LOGODDS are $1 / 5$ $>$ LOGODDS 2/10 > LOGODDS 3/15, etc. At the same time for the ratios $1 / 5,4 / 16$, and $7 / 27$, LOGGODS are equal. Therefore, we have the function that reflects the number of dissected LN. It would better reflect the number of LN dissected when the added factor was 0.75 or 1.15 , for example. On the other hand, there is no need to use logarithms for this purpose. It has no additional value for staging. Instead of LOGODDS one may use the ratio positive $L N+0.5 /$ negative $L N$ +0.5 in staging, and the results are the same. It is important to note that the LNR (probability), PNLNR (odds ratio), and log(PNLNR) all provide the same information.

\section{What is the difference?}

There is no difference in staging between LNR and PNLNR when the cutoffs correspond to one another. As an example, LNR cut-off 0.25 corresponds the value of 0.66 for PNLNR.

There is no correspondence of LNR (or PLNR) to LOGODDS because one LNR (PNLNR) has more than one value of LOGODDS. The reasons were clarified in the section above. The difference between LOGODDS results for a given value of $L N R$ (PLNR) depends on the difference between the number of LN examined [24].

The $\mathrm{pN}$ and LOGODDS staging relation is different when an increased number of $L N$ is examined (Figure 1). The cut-offs used by Sun et al. [23] and those published by Qiu et al. [25] differ mainly in less advanced stages. Stage $1-3$ by Sun et al. is equal to Stage 1 by Qiu et al. Stage 4 of the first classification is equal to Stage 2 of the second. For $6 \mathrm{LN}$ harvested there is no Stage 3 by Qiu. The classification proposed by Qiu et al. in stage 1 has in case of 16 LN harvested 0-5 metastatic LN and 
0-8 metastatic LN by 24 LN harvested. Therefore, we think it is more suitable for limited lymphadenectomy.

\section{Metastatic lymph nodes are not independent}

Lymph node status is an independent prognostic factor in gastric cancer almost regardless of the staging method. But there are other prognostic factors such as T stage, $M$ stage, vascular involvement, and histologic type of the tumour [26, 27]. N status is also related to other factors because different incidences of LN
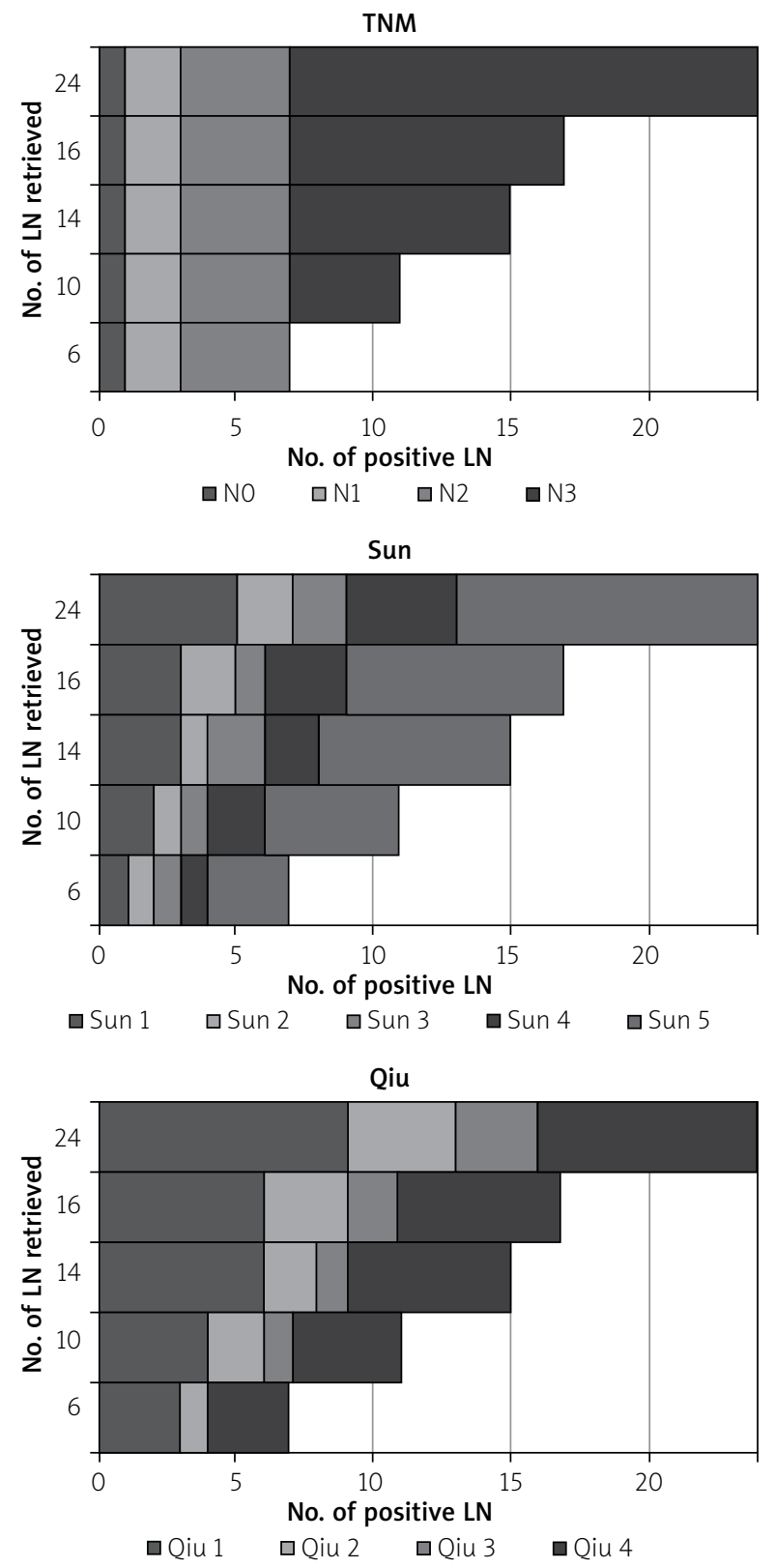

Figure 1. The comparison of TNM and LOGODDS staging metastases are observed in different T stages [27]. On the other hand, metastasis to the specific LN stations may be correlated with the poor prognosis [28]. Therefore, even with the most accurate classification of the $\mathrm{N}$ stage there is a possibility of subgroups with different survival within one $\mathrm{N}$ stage. Some other methods are implemented to predict individual survival, such as computer programs [29] or simpler nomograms [30] using more extensive data.

\section{Conclusions}

As the nature of gastric cancer is complex, it is probably not possible to predict the course of the disease with a single measure, which is $\mathrm{N}$ status. The additional confounding factor is the extent of lymphadenectomy and subsequent pathologic assessment of lymph nodes. The small number of examined lymph nodes produces low-quality data. Alternative systems such as LNR, PNLNR, and LOGODDS (or ODDS + 0.5) were implemented to increase the quality of $L N$ assessment. The main obstacle in the assessment of alternative staging methods is the lack of standard cutoffs for the stages. Another obstacle is the lack of common standards of lymphadenectomy. It is obvious that the level of dissection is different with 5-10 nodes harvested than with 25 nodes, even if it is called in both cases D2. The solution of this problem may be the common standard of staging for less than $16 \mathrm{LN}$, and another for 16 or more LN harvested. Some of the proposed methods provide the same information, and it is not surprising that the results are fully comparable. With LOGODDS the difference is made by the addition of a value of 0.5 to both to numerator and denominator, which itself is quite randomly selected among non integers. There is no proof that 0.5 is optimal for such an assessment.

\section{Conflict of interest}

The authors declare no conflict of interest.

\section{References}

1. Sobin LH, Gospodarowicz MK, Wittekind C (eds.). TNM Classification of Malignant Tumours. 7th edn., Wiley-Blackwell 2009.

2. Japanese Gastric Cancer Association. Japanese classification of gastric carcinoma: 3rd English edition. Gastric Cancer 2011; 14: 101-12.

3. Aurello P, D'Angelo F, Rossi S, et al. Classification of lymph node metastases from gastric cancer: comparison between $\mathrm{N}$-site and $\mathrm{N}$-number systems. Our experience and review of the literature. Am Surg 2007; 73: 359-66.

4. Reid-Lombardo KM, Gay G, Patel-Parekh L, et al.; Gastric Patient Care Evaluation Group from the Commission on Cancer. Treatment of gastric adenocarcinoma may differ among hospital types in the United States, a report from the National Cancer Data Base. J Gastrointest Surg 2007; 11: 410-9. 
5. Okines A, Verheji M, Allum W, et al. Gastric cancer: ESMO Clinical Practice Guidelines for diagnosis, treatment and follow-up. Ann Oncol 2010; 21 (Suppl. 5): v5054.

6. Kulig J, Popiela T, Kolodziejczyk P, et al. Polish Gastric Cancer Study Group. Standard D2 versus extended D2 (D2+) lymphadenectomy for gastric cancer: an interim safety analysis of a multicenter, randomized, clinical trial. Am J Surg 2007; 193 10-5.

7. Hundahl SA, Macdonald JS, Benedetti J, et al.; Southwest Oncology Group and the Gastric Intergroup. Surgical treatment variation in a prospective, randomized trial of chemoradiotherapy in gastric cancer: the effect of undertreatment. Ann Surg Oncol 2002; 9: 278-86.

8. National Clinical Audit Support Programme (NCASP). Leeds: The NHS Information Centre for health and social care; c2012. National oesophago-gastric cancer audit 2010. [Available at: http://www.ic.nhs.uk/services/national-clinicalaudit-support-programme-ncasp/audit-reports/ oesophago-gastric-cancer]

9. Ho VK, Damhuis RA, Hartgrink HH. Adherence to national guidelines for gastric cancer in the Netherlands: a retrospective population-based audit. Int J Cancer 2013; 132: 1156-61.

10. Lemmens VE, Dassen AE, van der Wurff AA, et al. Lymph node examination among patients with gastric cancer: variation between departments of pathology and prognostic impact of lymph node ratio. Eur J Surg Oncol 2011; 37: 488-96.

11. Espin F, Bianchi A, Llorca S, et al. Metastatic lymph node ratio versus number of metastatic lymph nodes as a prognostic factor in gastric cancer. Eur J Surg Oncol 2012; 38: 497-502.

12. Xu D, Huang Y, Geng Q, et al. Effect of lymph node number on survival of patients with lymph node-negative gastric cancer according to the 7th edition UICC TNM system. PLoS One 2012; 7: e38681.

13. Kwon SJ, Kim GS. Prognostic significance of lymph node metastasis in advanced carcinoma of the stomach. Br J Surg 1996; 83: 1600-3.

14. Kong SH, Lee HJ, Ahn HS, et al. Stage migration effect on survival in gastric cancer surgery with extended lymphadenectomy: the reappraisal of positive lymph node ratio as a proper N-staging. Ann Surg 2012; 255: 50-8.

15. Feinstein AR, Sosin DM, Wells CK. The Will Rogers phenomenon. Stage migration and new diagnostic techniques as a source of misleading statistics for survival in cancer. $\mathrm{N}$ Engl J Med 1985; 312: 1604-8.

16. Smith DD, Schwarz RR, Schwarz RE. Impact of total lymph node count on staging and survival after gastrectomy for gastric cancer: data from a large US-population database. J Clin Oncol 2005; 23: 7114-24

17. Liu C, Lu P, Lu Y, et al. Clinical implications of metastatic lymph node ratio in gastric cancer. BMC Cancer 2007; 7: 200.

18. Wang J, Dang P, Raut CP, et al. Comparison of a lymph node ratio-based staging system with the 7th AJCC system for gastric cancer: analysis of 18,043 patients from the SEER database. Ann Surg 2012; 255: 478-85.

19. Kulig J, Sierzega M, Kolodziejczyk P, et al. Polish Gastric Cancer StudyGroup. Ratio of metastatic to resected lymph nodes for prediction of survival in patients with inadequately staged gastric cancer. Br J Surg 2009; 96: 910-8.
20. Pedrazzani C, Sivins A, Ancans G, et al. Ratio between metastatic and examined lymph nodes ( $\mathrm{N}$ ratio) may have low clinical utility in gastric cancer patients treated by limited lymphadenectomy: results from a single-center experience of 526 patients. World J Surg 2010; 34: 85-91.

21. Lee SR, Kim HO, Son BH, et al. Prognostic significance of the metastatic lymph node ratio in patients with gastric cancer. World J Surg 2012; 36: 1096-101.

22. Huang CM, Lin JX, Zheng CH, et al. Prognostic impact of metastatic lymph node ratio on gastric cancer after curative distal gastrectomy. World J Gastroenterol 2010; 16: 2055-60.

23. Sun Z, Xu Y, Li de M, et al. Log odds of positive lymph nodes: a novel prognostic indicator superior to the number-based and the ratio-based $\mathrm{N}$ category for gastric cancer patients with $\mathrm{RO}$ resection. Cancer 2010; 116: 2571-80.

24. Xu J, Bian YH, Jin X, et al. Prognostic assessment of different metastatic lymph node staging methods for gastric cancer after D2 resection. World J Gastroenterol 2013; 19: 1975-83.

25. Qiu MZ, Qiu HJ, Wang ZQ, et al. The tumor-log odds of positive lymph nodes-metastasis staging system, a promising new staging system for gastric cancer after D2 resection in China. PLoS One 2012; 7: e31736.

26. Cammerer G, Formentini A, Karletshofer M, et al. Evaluation of important prognostic clinical and pathological factors in gastric cancer. Anticancer Res 2012; 32: 1839-42.

27. Brzozowa M, Michalski M, Harabin-Slowinska $M$, et al. The role of tumour microenvironment in gastric cancer angiogenesis. Prz Gastroenterol 2014; 9: 325-8.

28. Han KB, Jang YJ, Kim JH, et al. Clinical significance of the pattern of lymph node metastasis depending on the location of gastric cancer. J Gastric Cancer 2011; 11: 86-93.

29. Peeters KC, Hundahl SA, Kranenbarg EK, et al. Low Maruyama index surgery for gastric cancer: blinded reanalysis of the Dutch D1-D2 trial. World J Surg 2005; 29: 1576-84.

30. Han DS, Suh YS, Kong SH, et al. Nomogram predicting longterm survival after $\mathrm{d} 2$ gastrectomy for gastric cancer. J Clin Oncol 2012; 30: 3834-40.

Received: 25.06 .2015

Accepted: 18.01 .2016 